\title{
Télescope
}

Revue d'analyse comparée en administration publique

\section{Une famille recomposée : l'intégration d'un centre de réadaptation dans un centre hospitalier}

\section{Stéphanie Gagnon}

Volume 19, numéro 2, printemps 2013

L'innovation dans le secteur public : au-delà des discours

URI : https://id.erudit.org/iderudit/1023844ar

DOI : https://doi.org/10.7202/1023844ar

Aller au sommaire du numéro

Éditeur(s)

L'Observatoire de l'administration publique

ISSN

1929-3348 (numérique)

Découvrir la revue

Citer cet article

Gagnon, S. (2013). Une famille recomposée : l'intégration d'un centre de réadaptation dans un centre hospitalier. Télescope, 19(2), 113-129. https://doi.org/10.7202/1023844ar
Résumé de l'article

Cet article présente le cas de l'intégration de l'Hôpital Marie Enfant au CHU Sainte-Justine en 2000. Il s'agit d'un regroupement d'établissements de santé atypique par rapport à la majorité des fusions qui ont eu lieu dans le réseau de la santé québécois, d'une part parce qu'il a été volontaire (plutôt qu'imposé) et d'autre part parce qu'il concerne des clientèles spécialisées à différents moments du continuum de soins. L'accent est mis sur la gestion des frontières organisationnelles lors de la mise en oeuvre de l'intégration. Cette gestion se réalise grâce à des acteurs organisationnels différents selon la période à l'étude. Les résultats montrent que cinq types de frontières ont dû être gérés sur trois périodes pour mener à bien l'intégration. Le processus planifié a dû être modifié afin de gérer des frontières dont les enjeux n’ont pu être ignorés. 


\section{UNE FAMILLE RECOMPOSÉE : L'INTÉGRATION D'UN CENTRE DE RÉADAPTATION DANS UN CENTRE HOSPITALIER}

Par Stéphanie Gagnon, Professeure, École nationale d'administration publique stephanie.gagnon@enap.ca

RÉSUMÉ Cet article présente le cas de l'intégration de l'Hôpital Marie Enfant au CHU Sainte-Justine en 2000. II s'agit d'un regroupement d'établissements de santé atypique par rapport à la majorité des fusions qui ont eu lieu dans le réseau de la santé québécois, d'une part parce qu'il a été volontaire (plutôt qu'imposé) et d'autre part parce qu'il concerne des clientèles spécialisées à différents moments du continuum de soins. L'accent est mis sur la gestion des frontières organisationnelles lors de la mise en œuvre de l'intégration. Cette gestion se réalise grâce à des acteurs organisationnels différents selon la période à l'étude. Les résultats montrent que cinq types de frontières ont dû être gérés sur trois périodes pour mener à bien l'intégration. Le processus planifié a dû être modifié afin de gérer des frontières dont les enjeux n'ont pu être ignorés.

ABSTRACT This article presents the case of the integration of the hospital known as Hôpital Marie Enfant into the university medical centre known as CHU Sainte-Justine in 2000. In particular, this case concerns a merger of healthcare institutions that is atypical in comparison with most such mergers in the Quebec health system. For one, it was voluntary rather than imposed. Secondly, it involved specialized clienteles at different points along the care continuum. During the integration process, emphasis was placed on the management of organizational boundaries, to which various organizational actors contributed during the period under study. The results of the present study show that five types of boundaries had to be managed over three different periods in order to fully implement integration. The process, as originally planned, had to be modified in order to manage boundaries presenting issues requiring attention. 
Les fusions dans le secteur de la santé ont été nombreuses à partir des années 1990 dans plusieurs pays. En France, elles ont été considérées comme une solution à la rareté des ressources médicales (Forcioli, 2002), alors qu'au Royaume-Uni elles ont été associées au nouveau management public (Kitchener et Gask, 2003). Au Québec, elles ont eu lieu en deux vagues successives. Une première vague, poussée par un vent de rationalisation, s'est produite au début des années 1990 à la suite de la réforme Rochon et a essentiellement donné lieu à des fusions horizontales (fusions qui regroupent des établissements aux mandats similaires) et à la création de centres hospitaliers universitaires (CHU). La deuxième vague s'est amorcée au début des années 2000 et a mené à la création des centres de santé et de services sociaux (CSSS).

Le cas présenté dans cet article concerne l'intégration de l'Hôpital Marie Enfant (désormais Centre de réadaptation Marie Enfant - CRME) au CHU Sainte-Justine en 2000. Ce regroupement d'établissements est en rupture avec la majorité des fusions survenues dans le réseau de la santé québécois. D'une part, il était volontaire et non imposé et, d'autre part, il s'agissait d'un regroupement atypique puisqu'il n'était pas question de fusion horizontale, ni de multiregroupement dans le but de créer un CSSS. En fait, l'innovation est a priori issue de cette rupture avec la majorité des fusions. Nous verrons dans l'histoire de l'intégration que l'innovation se conçoit également par le processus déployé au fil du temps pour reconnaître et valoriser les différences entre les deux établissements étudiés.

Les fusions peuvent être considérées comme un moyen délibéré de modifier les frontières formelles des organisations (Denis, Lamothe et Langley, 2006). Or, d'après Hernes et Paulsen (2003, p. 3), les frontières organisationnelles auraient été étudiées uniquement de façon périphérique alors qu'elles ont leur propre dynamique. Selon Heracleous (2004), peu de travaux ont trait aux frontières en tant qu'entités complexes. Notre étude porte sur la réalité de la gestion des frontières à la suite de l'intégration de l'Hôpital Marie Enfant à l'Hôpital Sainte-Justine. Nous verrons que cinq types de frontières ont dû être considérés de façon séquentielle après cette intégration. Durant une première période, les activités ont porté sur la délimitation des frontières organisationnelles entre le CHU Sainte-Justine et l'hôpital de réadaptation (frontières structurelles et réglementaires), et entre la nouvelle entité (le CHU Sainte-Justine incluant le CRME) et les organisations du réseau de la santé (frontières relatives au pouvoir). Lors d'une deuxième période, les frontières identitaires de Marie Enfant par rapport au CHU Sainte-Justine ont été l'enjeu principal : leur gestion a impliqué la révision des frontières réglementaires tracées durant la première période. Finalement, lors d'une troisième période, la question des frontières relatives à l'identité a été consolidée, alors que la gestion des frontières relatives aux compétences et celles associées à l'influence ont été considérées entre le centre de réadaptation et son réseau d'appartenance. Nous constaterons que certains acteurs ont eu une influence prépondérante par leurs actions dans la gestion des frontières. La question à laquelle nous nous sommes intéressés est la suivante : comment les frontières organisationnelles sont-elles séquentiellement gérées lors d'un cas de fusion?

Nous présenterons le concept de frontières ainsi que ses différentes acceptions puisqu'il a servi à analyser les événements. Nous expliquerons par la suite nos choix méthodologiques pour ensuite tracer l'historique de l'intégration de l'Hôpital Marie Enfant à l'Hôpital Sainte-Justine à travers trois périodes : de l'intention à l'action (2000-2004), l'appropriation de l'intégration à l'interne (2004-2007) et l'intégration et ses retombées dans le réseau de la réadaptation (2007- ). Nous terminerons par une discussion sur ce qui se dégage des trois 
périodes décrites quant aux frontières et aux pratiques d'acteurs organisationnels dans leur gestion.

\section{UNE DIVERSITÉ DE FRONTIÈRES À GÉRER}

Plusieurs types de frontières sont reconnus dans la littérature. Par exemple, Denis et ses collègues (1999) suggèrent que le système de santé québécois a expérimenté des changements en fonction de quatre types de frontières : (1) les frontières intra-organisationnelles (structurelles et émergentes); (2) les frontières entre des organisations ayant des missions similaires; (3) les frontières entre des organisations ayant des missions différentes sur le continuum des soins; (4) les frontières du système de santé dans sa totalité. Les frontières intra-organisationnelles auxquelles ces auteurs font allusion peuvent référer à la division formelle des tâches (structurelles) ou aux frontières naturelles (émergentes) qui se créent dans la prestation de soins.

Hernes (2003), pour sa part, discute des frontières organisationnelles selon trois perspectives: physiques (matérielles et réglementaires), sociales et mentales. Les frontières matérielles ont trait à une matérialité au sens littéral du terme (les murs, par exemple), alors que les frontières réglementaires concernent la délimitation des interactions entre les acteurs organisationnels, leurs responsabilités et les attentes à leur égard. Ces dernières s'apparentent aux deux types de frontières intra-organisationnelles (structurelles et émergentes) proposées par Denis et ses collègues (1999). De leur côté, les frontières sociales permettent de différencier un groupe d'un autre et renvoient au concept d'identité. La littérature relative au lendemain des fusions accorde d'ailleurs une importance considérable au phénomène de l'identité (Langley et autres, 2012, p. 136 ${ }^{1}$ ). Quant aux frontières mentales, elles concernent « le répertoire particulier de termes et de symboles qui habilitent un groupe à communiquer, à agir et à approfondir leur compréhension du monde » (Hernes, 2003, p. 40).

Finalement, Santos et Eisenhardt (2005) proposent quatre conceptions de frontières associées à des enjeux organisationnels différents. Il s'agit des frontières relatives à l'efficience, celles associées à l'influence, celles qui se rapportent aux compétences et, enfin, celles de nature identitaire. La conception des frontières qui concerne l'efficience s'appuie sur la théorie des coûts de transaction. Il s'agit de minimiser ces coûts par la détermination stratégique des activités à internaliser et à externaliser. Le second type de frontières implique la sphère d'influence que l'organisation veut occuper dans un secteur d'activité donné. Il permet de rendre compte des arguments politiques (Comtois, Denis et Langley, 2004) évoqués pour justifier la décision de fusionner. Il s'agit alors de considérer la fusion comme un moyen qui contribue à la survie de l'organisation (maintien de son autonomie). Les frontières liées aux compétences ont trait aux ressources que les acteurs organisationnels choisiront d'investir dans la chaîne des valeurs de l'organisation. Enfin, les frontières identitaires impliquent une prise de position par rapport à «qui nous sommes». Elles sont l'équivalent des frontières sociales de Hernes.

Ces frontières sont présentées au tableau 1. Nous y suggérons des équivalences entre les diverses frontières proposées par les auteurs recensés et les classons essentiellement sous

1 Les traductions sont de l'auteure. 
quatre grandes catégories : individuelles, intra-organisationnelles, à la jonction des différentes organisations du système de santé et les frontières du système dans sa globalité.

Dans le domaine de la santé, certains travaux relatifs aux frontières se sont intéressés à leur redéfinition à la suite de changements dans le réseau de la santé (Denis et autres, 1999; Burri, 2008). Clark et ses collaborateurs (2010) ont traité de changements de troisième ordre, c'est-à-dire ceux qui concernent les frontières de plus d'une organisation. Ils ont montré que la présence d'une identité de transition facilitait la réalisation effective de ce type de changement. Enfin, d'autres travaux ont porté sur le travail frontalier requis quant aux frontières disciplinaires pour un fonctionnement optimal des équipes multidisciplinaires (Brown, Crawford et Darongkamas, 2000; Chreim et autres, 2013). Finalement, la gestion des frontières serait un défi demandant une attention particulière afin de maintenir l'identité et l'expertise organisationnelles.

\section{TABLEAU 1 : SYNTHÈSE DES TYPES DE FRONTIÈRES}

\begin{tabular}{|c|c|c|c|}
\hline $\begin{array}{l}\text { Catégories de } \\
\text { frontières }\end{array}$ & Denis et autres (1999) & Hernes (2003) & Santos et Eisenhardt (2005) \\
\hline $\begin{array}{l}\text { Frontières } \\
\text { individuelles }\end{array}$ & & Frontières mentales & \\
\hline $\begin{array}{l}\text { Frontières intra- } \\
\text { organisationnelles }\end{array}$ & $\begin{array}{l}\text { Structurelles } \\
\text { Émergentes: } \\
\text { communautés de } \\
\text { praticiens }\end{array}$ & $\begin{array}{l}\text { Matérielles } \\
\text { Réglementaires }\end{array}$ & \\
\hline \multirow{3}{*}{$\begin{array}{l}\text { Frontières à la } \\
\text { jonction des } \\
\text { différentes } \\
\text { organisations du } \\
\text { système de santé }\end{array}$} & $\begin{array}{l}\text { Frontières relatives à } \\
\text { des organisations } \\
\text { ayant des missions } \\
\text { similaires }\end{array}$ & & \\
\hline & $\begin{array}{l}\text { Frontières relatives à } \\
\text { des organisations } \\
\text { ayant des missions } \\
\text { différentes sur le } \\
\text { continuum des soins }\end{array}$ & & $\begin{array}{l}\text { Frontières relatives à } \\
\text { l'efficience }\end{array}$ \\
\hline & & Frontières sociales & Frontières identitaires \\
\hline $\begin{array}{l}\text { Frontières du } \\
\text { système dans sa } \\
\text { globalité }\end{array}$ & $\begin{array}{l}\text { Frontières du système } \\
\text { de santé }\end{array}$ & & $\begin{array}{l}\text { Frontières relatives au pouvoir, } \\
\text { à l'influence }{ }^{2} \text { : accroître sa } \\
\text { taille, réduire sa dépendance à } \\
\text { une seule activité, occuper } \\
\text { une position centrale dans un } \\
\text { réseau } \\
\text { Frontières associées à la } \\
\text { compétence : combinaison } \\
\text { différente de ressources }\end{array}$ \\
\hline
\end{tabular}

2 Les termes « frontières de pouvoir » et « frontières d'influence » seront utilisés en alternance dans le texte. 
Selon nous, la délimitation des frontières a trait à "l'identification des distinctions " (Marshall, 2003, p. 56). En fait, les frontières sont "produites et reproduites en créant des démarcations entre l'identité et la différence » (Marshall, 2003, p. 60).

\section{LA MÉTHODOLOGIE}

\section{L'étude de cas}

Nous avons réalisé une étude de cas afin de comprendre comment la gestion des frontières a progressé au fil du temps à la suite de l'intégration étudiée. De fait, l'étude de cas est une stratégie de recherche appropriée pour visualiser le processus et la dynamique des événements d'un point de vue longitudinal (Pettigrew, 1990).

Nous avons recueilli les données à partir d'entrevues semi-dirigées et d'une analyse documentaire. Quarante entrevues ont été menées auprès d'une pluralité d'acteurs, soit des médecins (6), des gestionnaires (14), des professionnels (14), des administrateurs (3), du personnel administratif (2) et un conseiller (1). Ces personnes ont été rencontrées à deux moments différents. Une première collecte de données a été effectuée auprès de 14 personnes en 1999-2000. Elle s'inscrivait dans un autre projet de recherche qui comparait deux changements stratégiques. Une seconde collecte a eu lieu auprès de 26 acteurs en 2007, 2008 et 2009 afin de connaître leur perception de l'intégration après un certain temps. Les individus ont été sélectionnés par un processus de recommandation de type snowball. Les entrevues ont permis de connaître la perception des acteurs quant à la réalisation de l'intégration et à sa raison d'être. Ces entretiens, dont la durée varie de 60 à 90 minutes, ont tous été transcrits. L'analyse documentaire est venue compléter l'information obtenue lors des entrevues et a permis de corroborer certains faits. Les documents utilisés concernaient spécifiquement l'intégration (protocole d'entente, suivi des transferts de clientèle, planification stratégique du programme de réadaptation) ou l'organisation (revue de presse, revue interne, etc.).

L'analyse des données a été réalisée à partir d'une description chronologique des événements. Nous avons ensuite mis en évidence les convergences et les divergences des propos des acteurs lors de séquences d'événements, ce qui nous a permis de cibler des phases critiques dans la réalisation de l'intégration. La stratégie de mise entre parenthèses du temps (traduction libre de temporal bracketing) (Langley, 1999) nous a permis d'utiliser les phases critiques pour déterminer des séquences de continuité et de discontinuité dans le processus d'intégration. Ce faisant, l'évolution des activités dans le temps est clairement ressortie, et il a été possible de saisir l'importance de certains événements sur le déroulement futur de ceux qui ont suivi. En agissant ainsi, les périodes sont devenues des unités d'analyse. Il a également été possible de les comparer entre elles, notamment par rapport aux types de frontières qui ont été gérés à différents moments.

\section{Les organisations à l'étude}

Le Centre de réadaptation Marie Enfant « offre des services spécialisés et surspécialisés d'adaptation-réadaptation, d'intégration et de participation sociales aux nouveau-nés, aux enfants et aux adolescents présentant une déficience motrice ou une déficience du langage » (Centre de réadaptation Marie Enfant, 2013). Le CHU Sainte-Justine, pour sa part, offre des soins de santé (spécialisés et ultraspécialisés) aux enfants, aux adolescents et aux mères du 
Québec. Ces deux hôpitaux prodiguent donc des soins qui varient d'un établissement à l'autre. Les acteurs qui y travaillent adoptent des philosophies d'intervention très différentes. Un chef de programme en témoigne :

Là-bas [Sainte-Justine], c'est une culture d'urgence, de première ligne. C'est vite, c'est la vie et la mort. C'est 365 jours sur trois quarts de travail... Tandis qu'ici [Marie Enfant], c'est long les processus de réadaptation. La réadaptation, c'est long en tant que tel. (Chef de programme, Centre de réadaptation Marie Enfant)

Le rôle des médecins diffère également d'un centre à l'autre. À Marie Enfant, ils font partie d'une équipe et constituent des acteurs parmi d'autres dans la prestation de services. Un médecin explique la dynamique qui prévaut à Marie Enfant :

C'est un endroit où on aide avec tous les moyens, parce qu'ici c'est rarement un médecin avec un enfant, c'est toujours une équipe. Alors qu'il y a peut-être des exceptions, en général le médecin est avec d'autres professionnels : physio, ergo... Il y aura toujours une réunion de professionnels pour faire un plan d'intervention personnalisé, pour dire « avec cet enfant-là, c'est ce plan-là qu'on prend, qui va davantage l'aider à devenir fonctionnel ». (Médecin, Centre de réadaptation Marie Enfant)

Finalement, ces deux établissements sont situés sur des sites distincts (éloignés géographiquement l'un de l'autre) et ils évoluent dans des réseaux différents, ce qui a des incidences sur les plans de la recherche, des partenaires et des orientations ministérielles. Ces deux hôpitaux relèvent de l'Agence de la santé et des services sociaux de Montréal, mais leurs activités interviennent à différents moments de la prestation de soins. De façon générale, les CSSS offrent des services de santé de première ligne, c'est-à-dire des soins de base, alors que les centres de réadaptation livrent des soins de deuxième ligne (soins spécialisés) ou de troisième ligne (soins ultraspécialisés). Pour avoir accès à ces services, une personne doit avoir été recommandée à la suite d'une consultation en première ligne. Les CHU prodiguent des services de troisième et de quatrième ligne. Ainsi, le CRME et le CHU Ste-Justine offrent des soins spécialisés à différents degrés. Le CHU évolue dans un environnement où la priorité est accordée aux soins curatifs, alors que le CRME travaille avec des partenaires en réadaptation, selon trois types de regroupements : la déficience motrice et la déficience du langage pour l'enfant; la déficience motrice et la déficience du langage pour l'adulte; la déficience sensorielle. Le CHU est membre de l'Association québécoise d'établissements de santé et de services sociaux. Le CRME, pour sa part, est membre de l'Association des établissements de réadaptation en déficience physique du Québec.

\section{L'INTÉGRATION ET SON DÉROULEMENT}

\section{De l'intention à l'action (2000-2004)}

Le rapprochement entre les deux établissements de santé n'est pas récent, puisqu'une coopération existe entre eux depuis plus de vingt ans. En fait, depuis le début des années 1980, l'Hôpital Sainte-Justine et l'Hôpital Marie Enfant collaboraient régulièrement (transfert des activités de réadaptation scolaire, recherche conjointe sur les meilleures prothèses pour les enfants, etc.). Puis, en 1995, les deux hôpitaux ont signé une entente de continuum de soins. Il s'agissait de :

[...] couloirs d'orientation de clientèles. Nous avions à identifier une personne-ressource qui était l'agent de liaison et l'Hôpital Sainte-Justine a fait la même chose, de façon à faciliter la transmission d'information. De part et d'autre, en ayant des agents de liaison, cela permettait de regrouper l'information pertinente et de s'organiser pour la transférer rapidement. Cela a vraiment accéléré le processus à Marie Enfant de mettre sur pied, par la présence de l'agent de liaison et l'implication du chef de programme, un mécanisme qui permettait de faciliter l'accès de la clientèle, d'éviter les duplica- 
tions quand l'information était pertinente pour déjà débuter l'intervention. (Chef de programme, Centre de réadaptation Marie Enfant)

Par la suite, une étude a été réalisée (par une firme indépendante) afin de formaliser davantage le regroupement. De cette étude a découlé, en avril 1999, un protocole d'entente qui a mené à l'intégration.

Le processus d'intégration a consisté à modifier le statut légal de l'Hôpital Marie Enfant en centre de réadaptation. Puis, le centre de réadaptation a été intégré à la structure par programmes de l'Hôpital Sainte-Justine. Le CRME devenait ainsi le neuvième programme de l'Hôpital Sainte-Justine ${ }^{3}$. Ce faisant, les postes administratifs associés aux ressources humaines, aux finances, etc. de Marie Enfant ont été abolis. Le fait que le Centre de réadaptation Marie Enfant devienne l'un des programmes de l'Hôpital Sainte-Justine a cependant eu pour conséquence de ne pas reconnaître les spécificités du centre de réadaptation. Un chef de programme témoigne :

[...] quand je dis one size fits all, c'est la loi de la majorité et c'est dans un hôpital, donc la réalité d'un hôpital et d'un centre de réadaptation c'est différent. Les politiques, les articles de convention, surtout dans le local, auraient sûrement mieux tenu compte de la réalité de notre secteur de réadaptation... Des fois, c'est désavantageux parce que ça ne tient pas compte des couleurs, des besoins particuliers. (Chef de programme, Centre de réadaptation Marie Enfant)

Par la suite (2002-2003), la directrice générale adjointe du CHU a veillé à ce que le centre de réadaptation soit représenté à l'Association des établissements de réadaptation en déficience physique du Québec. Les acteurs de Marie Enfant ont déploré certaines modifications apportées à leur quotidien à la suite de leur intégration d'un point de vue structurel, dont le sentiment de perdre de l'autonomie. Deux professionnels se prononcent :

\footnotetext{
Les gens avaient l'impression d'avoir perdu le sentiment d'autonomie. On avait toujours besoin de l'autorisation de Sainte-Justine. (Psychologue, Centre de réadaptation Marie Enfant)

On a l'impression qu'ils prennent les décisions et qu'on subit les décisions. Au niveau des orientations, par exemple, tel type d'enfant va être dans tel programme. On se dit : c'est qui, qui discute de ça? Est-ce qu'il y a eu du monde qui est venu nous voir? (Physiothérapeute, Centre de réadaptation Marie Enfant)
}

Les personnes ont par ailleurs noté un accroissement des procédures ainsi que des paliers hiérarchiques. Ces doléances sont intimement liées à la taille de Sainte-Justine par rapport à celle de Marie Enfant. Les affirmations suivantes sont éloquentes à ce sujet :

\footnotetext{
Marie Enfant, c'est comme un grain de sel dans un gros établissement. (Chef de programme, Centre de réadaptation Marie-Enfant)

Il y a eu ajout d'échelons, d'une superstructure... une surcharge de règlements administratifs... C'est plus régi... » (Psychologue, Centre de réadaptation Marie-Enfant)

Tout est compliqué! C'était beaucoup plus simple avant, il y avait moins d'intermédiaires. » (Coordonnatrice clinique, Centre de réadaptation Marie-Enfant)
}

De plus, les personnes interrogées font état de la perte de cadres directement accessibles sur le site de Marie Enfant. Par exemple, du point de vue administratif, la personne chargée des communications n'est présente que sur le site de Sainte-Justine tout comme le bureau de santé. Ce qui a pour conséquence de limiter la disponibilité en cas de besoin. Le chef professionnel est également moins présent à Marie Enfant puisqu'il passe l'essentiel de son

3 Les huit autres programmes étaient: mère-enfant, pédiatrie, multispécialités/transplantation, hématologieoncologie/immunologie-rhumatologie, soins intensifs/sciences cardiaques, urgence/traumatologie/chirurgie, sciences du développement et de la mobilité et psychiatrie. 
temps à Sainte-Justine. Les professionnels regrettent la perte de contact avec leur chef et estiment que cela nuit au développement professionnel ou la résolution de problème :

\begin{abstract}
Avant, il y avait un chef professionnel à Sainte-Justine et il y en avait un ici. Là, il n'y en a qu'un qui doit gérer à peu près 65 professionnels sur plusieurs sites. Ça peut vous donner une idée de la disponibilité qu'il a. Soit dit en passant, c'est quelqu'un qui a une énergie et qui met du sien à $150 \%$, mais c'est comme humainement parlant pas faisable. Si on a un rendez-vous avec lui, il va l'annuler au moins deux fois sur trois, pour des raisons qu'il peut justifier. Mais il reste que nous, à la base, on passe tout le temps notre tour, et ça, ça amène de la démotivation. (Professionnelle, Centre de réadaptation Marie Enfant)
\end{abstract}

Il ressort de cette première période que les frontières qui ont été gérées concernaient essentiellement l'intra-organisationnel. En effet, l'une des premières actions prises a consisté à gérer les frontières structurelles en faisant de l'établissement Marie Enfant un programme de l'Hôpital Sainte-Justine. Il s'agissait d'intégrer structurellement Marie Enfant au sein de l'organigramme de Sainte-Justine. Puis, les frontières réglementaires ont amené des procédures standardisées au Centre de réadaptation Marie Enfant, créant pour ses acteurs un sentiment de perte d'autonomie.

Les frontières d'influence ont également été traitées puisque le fait de rejoindre l'Association des établissements de réadaptation en déficience physique du Québec permettait à la nouvelle entité (le CHU Sainte-Justine intégrant le Centre de réadaptation Marie Enfant) d'être reconnue comme un acteur dans le domaine de la réadaptation.

Du point de vue de l'intention associée à la décision d'intégration, ce sont surtout les frontières du système de santé qui étaient visées. De fait, l'Hôpital Sainte-Justine obtenait un continuum de soins avec l'intégration, comme l'explique un membre du conseil d'administration de cet établissement :

Quand on nous a nommés centre hospitalier universitaire, la réadaptation devenait naturelle. À ce moment-là, on pouvait investir en réadaptation, ce qu'on a fait d'ailleurs. [...] Notre clientèle apprécie beaucoup de savoir que si elle a un accident et un problème de mobilité, elle vient ici [Sainte-Justine]. Son enfant est traité ici puis après ça, ils s'en vont à Marie Enfant. Il y a un continuum, le patient est suivi par les mêmes équipes. (Membre du conseil d'administration, CHU Sainte-Justine)

Ce faisant, cet établissement de santé cherchait à démontrer son influence dans le réseau de la santé en affichant une position enviable. Par ailleurs, le rapprochement entre les deux établissements a également eu un effet sur l'obtention de masses critiques. Un pédiatre témoigne de ce fait :

C'était toujours le principe : on est petit, il faut grossir. On n'est pas si fort que ça... Et donc, essayons d'augmenter la masse critique de l'Hôpital [...]. L'Hôpital s'est beaucoup consolidé avec ça et a ajouté un volet très important. (Pédiatre, CHU Sainte-Justine)

Cette stratégie supposait aussi de modifier les frontières relatives à la compétence. De fait, l'obtention d'une masse critique est associée à l'atteinte de l'excellence dans l'acte. De plus, de nouvelles ressources étaient ajoutées dans l'offre de soins (ressources en réadaptation). Pour l'Hôpital Marie Enfant, l'intégration rendait possible l'obtention du statut universitaire $^{4}$. Un pédiatre qui travaille à la fois à Sainte-Justine et à Marie Enfant nous a expliqué que l'Hôpital Marie Enfant avait déjà demandé, en 1990 en commission parlementaire, à être reconnu comme institut universitaire. À ce moment, la démarche n'avait pas porté ses fruits. L'Hôpital Marie Enfant cherchait alors à modifier les frontières d'influence en démontrant ses habiletés et ses ressources dans le domaine de la recherche.

4 Dans la collecte de données des années 2007 à 2009, ce n'est pas le message qui est principalement véhiculé. 


\title{
L'appropriation de l'intégration à l'interne (2004-2007)
}

Les insatisfactions mentionnées précédemment ont entraîné une détérioration du climat de travail dans les premières années suivant l'intégration. Quatre ans après, la haute direction de Sainte-Justine est intervenue pour recueillir les doléances des gens, ce qui a donné lieu à une diversité d'actions pour améliorer la situation. Parmi celles qui se sont avérées significatives, figure la réouverture de la cafétéria, laquelle a suscité un réel enthousiasme :

\begin{abstract}
Je me souviens de l'abolition de la cafétéria qui avait eu lieu en 2000 et qui avait fait une cassure [...] On a procédé, peu de temps après que j'arrive [2004] à l'inauguration du casse-croûte ici, qui était un peu comme le symbole du redémarrage de l'entité de Marie Enfant. C'était le lieu de rassemblement des gens qui étaient là le midi, il y avait une concession qu'on avait engagée et c'était très symbolique. (Chef de programme, Centre de réadaptation Marie Enfant)
\end{abstract}

La direction est également intervenue dans la signature de l’Hôpital Marie Enfant. Celleci est demeurée distincte. Une gestionnaire commente :

L'autre chose qui... des fois ça peut paraître anodin, c'était sur l'insigne en avant, la pancarte, c'était «CHU Sainte-Justine Centre de réadaptation Marie Enfant». Personne ne le prenait. « On n'est pas SainteJustine! On est le Centre de réadaptation Marie Enfant! » Puis, ça n'a pas été long pour que madame Turcotte ${ }^{5}$ fasse inverser ça : « Centre de réadaptation Marie Enfant du CHU Sainte-Justine ». (Gestionnaire, Centre de réadaptation Marie-Enfant)

Par la suite, quelques acteurs ont contribué au changement de certaines pratiques, notamment en misant sur la dénomination "Centre de réadaptation » (avant l'intégration, il s'agissait de l'«Hôpital» Marie Enfant). Cette appellation implique une philosophie d'intervention particulière, et la façon de mener les activités de réadaptation est actuellement en mutation. " D'une perspective de compassion, la réadaptation a évolué vers celle d'une intégration et d'une participation sociale de la personne handicapée, reflétant un changement majeur au sein de notre société » (Samson Saulnier, 2007, p. 3). Une coordonnatrice clinique commente ce changement à l'interne :

\footnotetext{
Il y a déjà plein de choses qui ont changé au niveau du fonctionnement. Exemple, juste au niveau du plan d'intervention... Moi, j'étais habituée à en faire souvent, et quand je suis arrivée ici et que j'ai vu la façon dont c'était fait... Je disais « ce n'est pas comme ça là ». Et là, on est venu à la philosophie de la réadaptation : concerter le parent, s'asseoir et regarder ça ensemble... Je trouve qu'en trois ans, il y a des choses qui ont vraiment évolué. On est plus proche de ce qu'est la réadaptation. (Coordonnatrice clinique, Centre de réadaptation Marie-Enfant)
}

Ces changements dans la philosophie d'intervention sont aussi le reflet d'autres événements qui touchent le centre de réadaptation. Une gestionnaire raconte :

\begin{abstract}
Aujourd'hui, ce sont la transformation du Réseau, les nouvelles lignes directrices ministérielles et la volonté de développer une culture d'évaluation de programmes [...] qui nous amènent à nous engager dans une révision en profondeur des programmes de l'établissement. (Vaillancourt, 2005)
\end{abstract}

Le temps a aussi fait son œuvre dans la mesure où certains mécanismes de fonctionnement se sont installés entre Sainte-Justine et Marie Enfant. Des bénéfices sont manifestes. Enfin, l'annulation de certaines politiques élaborées par Sainte-Justine pour les deux établissements a contribué à assainir le climat. Ce type d'action permet de reconnaître le caractère distinct de la réadaptation par rapport aux soins de courte durée. Les témoignages suivants confirment ces faits :

5 Tous les noms sont des pseudonymes. 


\begin{abstract}
Pour les consultations externes (en neurologie, en pédopsychiatrie, etc.), ça aide effectivement, en termes d'accessibilité. Ça aide les enfants à avoir des services plus rapidement. (Coordonnatrice clinique, Centre de réadaptation Marie-Enfant)

L'exemple qui me vient en tête, c'est il y a un an ou deux, ils avaient fait une politique de tenue vestimentaire. Est-ce que je suis la première qui vous en parle? (Rires). Je suis parfaitement d'accord, il faut s'habiller comme du monde, convenablement pour venir travailler. Mais on n'est pas dans un milieu de microbes comme Sainte-Justine. Ça prend des mesures de base, oui se laver les mains, s'il y a un enfant qui te crache dessus, c'est le fun d'avoir un petit t-shirt dans ton tiroir là. Mais de là à la sortir de façon... Ça a provoqué presque à la fois des fous rires puis de la colère ici... des choses qui ne s'appliquaient pas. Il y a eu un petit revirement et ils ont demandé : «Qu'est-ce que vous modifieriez? Comment peut-on l'adapter à Marie Enfant? »C'est retourné aux grandes instances qui devaient repenser une version Marie Enfant, et on n'en a jamais réentendu parler. (Chef de programme, Centre de réadaptation Marie Enfant)
\end{abstract}

Au cours de cette seconde période, on observe des actions essentiellement axées sur les frontières identitaires. La Direction de Sainte-Justine reconnaît certaines spécificités aux programmes de Marie Enfant, dont l'acceptation de pratiques vestimentaires différentes. Dans le même ordre d'idées, la signature distincte est reconnue. Ces actions confirment que Marie Enfant est différent de Sainte-Justine.

L'action d'annuler la politique vestimentaire s'inscrit aussi sous la frontière réglementaire. Il s'agit d'un retour en arrière par rapport à la standardisation des politiques prônée à la période précédente. Les acteurs de Marie Enfant proposent par ailleurs l'homogénéisation à l'intérieur des programmes ${ }^{6}$ de leur établissement. Ils ont rédigé le Guide d'élaboration des programmes en déficience physique du CRME. Une chef de programme explique :

\footnotetext{
Je réalisais, dans ma première année, que chacun des programmes travaillait en vase clos. Chaque chef de programme décidait que telle chose c'est non, telle chose c'est permis. Puis le contraire, dans l'autre programme. Donc, si un intervenant est sur la liste de rappel, puis change de poste, il voyait que dans ce programme-là tout le monde était d'accord avec telle optique pis là-bas, ça marchait pas comme ça. Je disais toujours : « Il faut se concerter, il faut tous être pareils, on est dans la même boîte. » Les programmes clientèles doivent utiliser les mêmes outils, réfléchir de la même façon, penser les mêmes normes de gestion... Je l'ai fait pour mon département, mais j'ai poussé les gens pour que ça se fasse tous en même temps. (Chef de programme, Centre de réadaptation Marie Enfant)
}

Les acteurs de Marie Enfant s'arriment également davantage à la philosophie de la réadaptation prônée dans la société, et ce, en conséquence du nouveau statut de l'établissement et d'événements extérieurs qui les concernent. Ainsi, ce centre de réadaptation consolide ses ressemblances avec le réseau de la réadaptation. Les actions posées lors de cette période illustrent la tension entre les similitudes (avec le réseau de la réadaptation et les autres programmes du CRME) et les différences (avec le CHU Sainte-Justine et les soins de courte durée) relatives à la question identitaire lors de fusions (Langley et autres, 2012).

\title{
L'intégration et ses retombées dans le réseau de la réadaptation (2007- )
}

Les activités internes au CRME visant à faciliter l'intégration se sont poursuivies au cours des années subséquentes. Les gains réalisés pour des pratiques différenciées par rapport à Sainte-Justine sont commentés par un participant à l'étude :

6 Les activités du CRME sont elles aussi découpées selon les programmes offerts à la clientèle : maladies neuromusculaires, déficits moteurs cérébraux, retards de développement, amputés/musculosquelettiques et neurotraumatologie, réadaptation en milieu scolaire, réadaptation fonctionnelle intensive, réadaptation de la parole et du langage, ressources résidentielles. 
On a l'impression qu'ils nous donnent les outils pour revendiquer nos besoins spécifiques. [...] Récemment, il y a eu la mise en place d'un comité de main-d'œuvre Marie Enfant, d'un comité académique Marie Enfant. Et ça, c'était urgent parce que justement, les enjeux et les problématiques qu'on vit ici ne sont pas les mêmes qu'à Sainte-Justine. (Chef de programme, Centre de réadaptation Marie Enfant)

On constate par ailleurs que de plus en plus d'actions sont orientées vers l'externe, particulièrement vers le réseau de la réadaptation. De fait, le centre de réadaptation intégré au CHU Sainte-Justine évolue dans un réseau qui est différent. Un médecin témoigne :

On veut intégrer des choses, mais vous savez maintenant il y a un réseau universitaire intégré de santé qui est avec les centres hospitaliers et les CSSS, et en parallèle, il y a un deuxième réseau qui est le réseau de la réadaptation, qui n'a pas les mêmes structures, qui n'a pas les mêmes territoires, qui n'a pas les mêmes organisations... Alors moi si je donne congé à un patient du centre Marie Enfant, il ne s'en va pas dans un autre hôpital, il s'en va dans le réseau de réadaptation. Il va s'en aller au Bouclier ou au centre de réadaptation Laurentides, donc c'est un réseau différent. [...] C'est plus difficile, la résistance, elle n'est pas juste à l'interne, l'environnement n'est pas facilitant. (Médecin, CHU Sainte-Justine)

En ce qui concerne cette réalité, le rayonnement des activités conduites au centre de réadaptation est parfois plus difficile. Un chef de programme explique :

Sur le plan de la recherche, comme on est intégré à Sainte-Justine, il a fallu faire une entente avec le CRIR [Centre de recherche interdisciplinaire en réadaptation]. Parce qu'il y a un réseau de recherche en réadaptation pour lequel les intervenants, disent: «Bien, écoute, je ne peux pas être membre comme chercheur de ce réseau-là parce qu'ils me disent que je suis affilié à Sainte-Justine. » Puis, ils trouvent ça dur : «Voyons donc, ça n'a pas d'allure de ne pas faire partie de ce réseau-là... » La diffusion de ce qu'on fait est plus laborieuse et on a l'impression des fois d'être déconnectés du réseau de la réadaptation. (Chef de programme, Centre de réadaptation Marie Enfant)

Ainsi, quelques actions ont été menées de 2007 à 2009 avec pour objectif de formaliser certaines différences entre Marie Enfant et Sainte-Justine. Cependant, l'appartenance au réseau de la réadaptation semble devoir être consolidée de façon à accroître le rayonnement de Marie Enfant en son sein. Diverses démarches ont donc été entreprises à cet égard. Par exemple, dès 2007, le journal du CRME, Le Capteur d'idées ${ }^{7}$, incluait dans ses premières pages un descriptif du CRME relativement à sa mission, à son équipe et à son importance du point de vue de la réadaptation. Dans les éditions antérieures, cette information n'y figurait pas. La même année, le Centre s'associait à dix autres centres de réadaptation québécois et européens afin de former le Réseau francophone en déficience sensorielle et du langage.

L'année suivante, en décembre, le nouveau site Internet du CRME était lancé. Dans la présentation sommaire du site, l'accent est mis sur la famille de l'enfant et sur les professionnels. Le dernier paragraphe du communiqué de presse annonçant le site est intitulé Une ouverture sur le milieu de la réadaptation pédiatrique. Le site a d'ailleurs été récompensé par l'Association des établissements de réadaptation en déficience physique du Québec dans la catégorie «Communication ». Il semble que ces démarches aient été nécessaires parce qu'en 2008 le CRME n'était toujours pas reconnu de manière généralisée comme centre de réadaptation dans le réseau de la santé :

J'avais une rencontre la semaine dernière avec le Montreal Children pour leur demander qui diagnostiquait un certain type de maladie. Pour dire ensuite : «Écoutez, le Ministère a imposé un cadre des services surspécialisés parce qu'il considérait que ces clientèles-là ont besoin d'un suivi. Donc, il faut que vous les référiez en réadaptation pour qu'on puisse répondre à ces besoins-là. ». Et là, ils disaient: «Bien non, c'est parce que nous, on en a une telle clinique.» « Oui, je suis d'accord. Mais nous, on est un centre de réadaptation. » «Oui, mais tes médecins, ils viennent de Sainte-Justine.» Et là, je rectifiais : «Oui, ils peuvent travailler à Sainte-Justine dans un mandat X, mais moi ici, quand ils travaillent à Marie

$7 \quad$ Le Capteur d'idées est publié semestriellement et sa première édition est parue en 2005. 
Enfant c'est dans un mandat de réadaptation. » Et ça ne passait pas. Le Children ne nous voyait pas comme un centre de réadaptation, il nous voyait comme une annexe de Sainte-Justine. (Chef de programme, Centre de réadaptation Marie Enfant)

En 2009, le nom de la fondation du CRME a été revu. Elle se nomme désormais Fondation Mélio, dont la racine latine signifie meilleur. «C'est l'origine du mot "amélioré", rendre meilleur » (Centre de réadaptation Marie Enfant, 2009). Un cadre explique le maintien ${ }^{8}$ de deux fondations séparées :

Parce qu'on disait : «On n'a pas le même public cible, on n'a pas les mêmes objectifs, puis si on est intégré là-bas, on ne verra peut-être pas la couleur de l'argent qu'ils reçoivent à Sainte-Justine à la Fondation. Nous, on veut faire nos projets, monter nos choses. » Puis on l'a eue, on a une nouvelle fondation, notre image, qui est là pour rester. Puis, je pense que c'est important. On disait intégration, mais finalement c'est important de garder de petits éléments de culture de l'organisation qui sont propres, qui nous ressemblent, qui répondent à ce qu'on a besoin. (Cadre, Centre de réadaptation Marie Enfant)

En 2011, la Chaire de recherche en génie de la réadaptation pédiatrique « a été cofondée par l'École Polytechnique de Montréal et le CRME » (Raison et autres, 2011, p. 12). Ce qui transparaît au cours de cette troisième période est la place centrale qu'occupe la question identitaire. La différenciation se poursuit par rapport au CHU Sainte-Justine et s'exprime à la fois à l'interne (comité distinct) et à l'externe (Fondation Mélio). Toutefois, la question de l'externe prend de l'ampleur et les acteurs du CRME sont proactifs pour se faire connaître dans le réseau de la réadaptation. Il semble y avoir une appropriation de cette préoccupation par les acteurs de Marie Enfant, alors que les activités de ce type étaient auparavant menées par la directrice générale adjointe du CHU Sainte-Justine. Ces acteurs se comparent avec des établissements du réseau de la réadaptation et ils conçoivent que la pression soit forte pour rayonner. Leurs actions portent à la fois sur les frontières d'influence (reconnaissance, partenariat) et de compétence (déploiement d'une chaire de recherche). Une chef de programme et une coordonnatrice commentent l'importance de se faire reconnaître :

\begin{abstract}
Oui. Tu sais des fois quand tu grattes, tu te dis qu'on fait la même chose, mais ils sont capables de se mettre en valeur, soit par des petites formations... Je dirais que là-dessus on a peut-être un petit retard, mais on a notre place aussi là. Je dirais qu'il faut que les équipes sortent de la morosité, là je pense qu'on est en train de sortir... (Chef de programme, Centre de réadaptation Marie-Enfant)

Ce qu'on voit, c'est que le centre Cardinal-Villeneuve à Québec a pris beaucoup d'essor, que le centre montérégien de réadaptation, du côté de la Rive-Sud, a pris beaucoup d'essor aussi. Et ce qu'on a l'impression à Marie Enfant, c'est qu'il y a toujours un remodelage, continuellement! Ce qui fait qu'on n'arrive pas à avancer, et que dès qu'il y a un peu de temps pour dire " ah ben on va pouvoir créer un projet, faire quelque chose, partir quelque chose ", là c'est une coupure qui arrive. (Coordonnatrice, Centre de réadaptation Marie Enfant)
\end{abstract}

De plus, l'afflux de clientèle est différent par rapport au passé en raison de la régionalisation récente. Une professionnelle explique l'effet sur l'organisation du travail et, par conséquent, sur la nécessité de se coordonner avec les autres acteurs de la réadaptation :

Nous sommes une clinique surspécialisée en maladies neuromusculaires, ce qui fait qu'on demeure les experts pour répondre aux gens des régions, mais ces clients doivent être suivis en spécialité par les thérapeutes des régions. C'est sûr que maintenant on va les voir juste sur la demande d'un intervenant en région ou d'un parent, ou lorsqu'ils viennent d'une clinique médicale ici. Alors on les voit moins, ce qui fait qu'on voit moins de clients. C'est tout le passage, la transition qui commence à s'opérer. (Professionnelle, Centre de réadaptation Marie Enfant)

Ainsi, au cours de cette troisième période, les changements concernent plutôt l'appartenance, au-delà du CHU, à un réseau distinct. Les activités s'inscrivent dans la né-

8 La décision de maintenir deux fondations distinctes pour chacun des établissements de santé a été prise lors du protocole d'entente de 1999. 
cessité de se différencier (frontière de compétence), de rayonner à l'externe et de créer des partenariats (frontière d'influence). Cela semble possible après avoir bien distingué les acteurs organisationnels de Marie Enfant (frontières identitaires) de ceux de Sainte-Justine.

\section{DISCUSSION ET CONCLUSION}

Les résultats précédents tendent à démontrer que la gestion des frontières organisationnelles lors d'un cas de fusion se réalise graduellement, passant des frontières intraorganisationnelles aux frontières identitaires, pour se terminer par les frontières relatives à la compétence et à l'influence. De plus, les acteurs influents dans cette gestion augmentent avec le temps, à mesure qu'ils se reconnaissent dans l'organisation recréée. Finalement, la gestion des frontières suppose de s'occuper de sphères inattendues.

\section{Un ordonnancement particulier des frontières à gérer}

La gestion des frontières à travers ces trois périodes montre que l'ordonnancement des frontières à traiter est important. Lors d'une première étape, les frontières structurelles et réglementaires sont gérées. Les premières sont définitivement réglées à ce moment-là. Cela est conforme aux résultats et aux pratiques recensés dans des études similaires (Denis et autres, 2009, p. 243) selon lesquelles « la structure joue un rôle central dans la réduction de l'ambiguïté ». Durant cette même période, les frontières d'influence sont également abordées. Cela est comparable aux objectifs poursuivis dans de multiples cas de fusions en santé (Comtois, Denis et Langley, 2004) dans le but de projeter un statut prestigieux.

La seconde étape amène plutôt à gérer les frontières identitaires qui avaient d'abord été négligées. Les préoccupations associées à ces frontières émergent à la suite des actions entreprises au cours de la première période. Quoique les enjeux sous-jacents aux préoccupations identitaires soient normaux et fréquents lors de fusions (Denis, Lamothe, Langley, 1999; Clark et autres, 2010), ils n'avaient pas été anticipés. De fait, les questions identitaires sont nombreuses dans ce contexte : sentiment de perte de contrôle, menaces perçues, perte de confiance, luttes, etc. (Fulop et autres, 2005; Clark et autres, 2010; Langley et autres, 2012).

La seconde période a donc touché les questions identitaires et, en corollaire, les frontières réglementaires. Or, d'après Alvesson, Ashcraft et Thomas (2008), l'identité peut également se refléter dans les pratiques et les dispositions matérielles. La gestion des frontières réglementaires par l'intermédiaire de la politique vestimentaire apparaît donc étroitement liée à la question identitaire.

Enfin, au cours de la troisième période, la question des frontières identitaires demeure importante, mais en voie de résolution. En effet, certaines actions offrent une marge de manœuvre aux acteurs de Marie Enfant quant au recrutement du personnel (comité de main-d'œuvre) et à la fondation. Selon Langley et ses collègues (2012), l'idée d'offrir des zones où les différences sont tolérées entre des établissements fusionnés aide à susciter l'engagement envers le nouvel établissement. De plus, le rayonnement et l'ouverture vers le réseau de réadaptation semblent pointer vers une démonstration de « qui nous sommes » à l'extérieur du CHU et une volonté d'être actif dans ce réseau. Les frontières d'influence et de compétence sont utilisées pour montrer l'offre de services, identifier les partenaires et protéger sa sphère d'influence. 


\section{Une variété d'acteurs impliqués}

Les acteurs qui contribuent à faciliter l'intégration varient dans le temps et sont surtout plus nombreux à partir de la deuxième période. Lors de la première période, la directrice générale adjointe du CHU a joué un rôle prépondérant lorsqu'elle a veillé à ce que le CRME soit présent à l'Association des établissements de réadaptation en déficience physique du Québec. Elle s'est d'ailleurs très rapidement jointe au conseil d'administration de l'Association, faisant ainsi connaître davantage l'établissement Marie Enfant dans le réseau de la réadaptation. Dans le passé, les acteurs de Marie Enfant étaient peu enclins à s'ouvrir à leur réseau : Une gestionnaire commente :

\footnotetext{
Quand je suis arrivée à Marie Enfant [années 1990], ma perception à ce moment-là, c'était un peu comme si c'était un monde élitiste. C'étaient des gens qui étaient très compétents, qui avaient une expertise en réadaptation pédiatrique, mais un peu comme repliés sur eux-mêmes par rapport aux partenariats, aux centres régionaux, aux référents. (Gestionnaire, Centre de réadaptation Marie Enfant)
}

Les doléances exprimées durant cette période par les acteurs organisationnels de Marie Enfant sont liées à l'identité qu'ils avaient avant l'intégration. Ce facteur ainsi que des récriminations contre le nouveau partenaire sont sources d'inertie d'après Clark et ses collègues (2010). Cela permet de comprendre l'inaction des acteurs organisationnels de Marie Enfant à ce moment-là.

Puis, de 2004 à 2007, la directrice générale adjointe a cherché à assainir le climat à l'interne. En parallèle, trois chefs de programme ont eu une influence importante sur les événements subséquents. L'une d'entre elles s'est efforcée d'harmoniser les pratiques de gestion des programmes de réadaptation. Une autre a contribué à la révision des programmes à l'interne, alors qu'une troisième a œuvré auprès de son équipe pour faire adopter la philosophie de réadaptation. La pluralité d'acteurs qui influencent le cours des événements est conforme aux travaux en santé qui reconnaissent le leadership distribué dans ce domaine (Chreim et autres, 2010). De même, les acteurs qui interviennent le font dans des sphères différentes. La directrice générale adjointe agit du point de vue de l'administratif, alors que les chefs de programme œuvrent à la jonction du clinique et de l'administratif. Leur plus grand nombre apparaît refléter une appropriation de ce qu'est dorénavant le Centre de réadaptation Marie Enfant.

Enfin, durant la troisième période, un certain nombre d'acteurs organisationnels ont contribué à faire rayonner le CRME. Les acteurs impliqués sont encore plus diversifiés qu'aux étapes précédentes. La directrice générale adjointe du CHU Sainte-Justine est présente lors de la signature du protocole du Réseau francophone en déficience sensorielle et du langage, et de nouveaux acteurs interviennent. Les administrateurs de la fondation du CRME jouent un rôle dans le repositionnement stratégique de la fondation, alors que les médecins participent à la création d'une chaire universitaire.

\section{Une gestion à la fois planifiée et émergente des frontières}

L'histoire de l'intégration de l'Hôpital Marie Enfant à l'Hôpital Sainte-Justine montre que malgré plusieurs actions planifiées certains événements émergents ont une influence considérable sur les actions futures. Ainsi, les actions menées au cours de la première période sont essentiellement de nature planifiée. Par exemple, l'idée de faire de l'établissement Marie Enfant un neuvième programme était prévue dans un document intitulé Projet de consolidation, de développement et de promotion des services pédiatriques HSJ/HME, et ce, dès février 1999. Durant la seconde période, deux événements imprévus ont grandement influen- 
cé la suite du processus d'intégration. Il s'agit de la question des frontières identitaires et des changements en réadaptation instaurés par le ministère de la Santé et des Services sociaux. Ces deux faits ont influé considérablement sur les activités et les choix futurs. D'une part, les acteurs de Marie Enfant ont démontré leurs différences et les ont fait accepter au sein de l'établissement Sainte-Justine, d'autre part, ils ont mis en place un certain nombre d'activités qui les ont rapprochés du milieu de la réadaptation. Enfin, à la troisième période, les actions ont été à la fois planifiées et émergentes, et elles ont contribué à consolider la place de Marie Enfant dans le réseau de la réadaptation.

Finalement, un horizon temporel important, près de dix ans, aura été nécessaire pour réaliser les objectifs poursuivis par l'intégration. La gestion des frontières d'influence et de compétence a mené les établissements vers l'atteinte des objectifs définis au cours de la troisième période. De fait, tant et aussi longtemps que les questions identitaires n'étaient pas résolues, il était difficile de penser à se faire connaître et reconnaître à la fois comme CHU distinct (volonté de Sainte-Justine) et comme centre de réadaptation (Marie Enfant).

Nos résultats montrent qu'à contrario de plusieurs études sur les fusions, ce ne sont pas les frontières internes qui constituent un défi. Dans le cas présenté, c'est plutôt la résolution des frontières identitaires qui a permis de s'intéresser subséquemment aux frontières relatives au réseau de la réadaptation. L'acceptation de deux identités distinctes a permis aux acteurs organisationnels de miser sur leurs spécificités et de se préparer à les faire connaître à l'externe. L'attention séquentielle portée aux frontières s'est avérée une stratégie efficace même si elle n'était, à priori, pas anticipée. La redéfinition des frontières à l'interne et la gestion des frontières avec l'externe ont donné lieu à une révision des rôles entre acteurs de Sainte-Justine et de Marie Enfant, démontrant le processus d'appropriation de l'intégration pour les acteurs de Marie Enfant.

L'innovation produite dans cette étude de cas concerne ainsi à la fois le résultat final et le processus déployé pour y arriver. Autrement dit, la réalité d'un centre de réadaptation intégré dans un CHU de soins aigus, qui fonctionne et cherche à accroître sa légitimité, constitue, en soi, une innovation. Celle-ci prend, par ailleurs, tout son sens dans le nouvel équilibre qui a été créé dans les actions du CHU par rapport au CRME. Nos conclusions montrent que l'acceptation des différences entre les deux établissements a été déterminante dans l'issue du processus.

\section{BIBLIOGRAPHIE}

Alvesson, M., K. L. Ashcraft et R. Thomas (2008). «Identity Matters: Reflections on the Construction of Identity Scholarship in Organization Studies », Organization, vol. 15, n 1 , p. 5-28.

Brown, B., P. Crawford et J. Darongkamas (2000). «Blurred Roles And Permeable Boundaries: The Experience Of Interdisciplinary Team Working For Community Mental Health Staff' », Health and Social Care in the Community, vol. 8, no 6, p. 425-435.

Burri, R. V. (2008). "Doing Distinctions: Boundary Work and Symbolic Capital in Radiology», Social Studies of Science, vol. 38, no 1, p. 35-62.

Chreim, S. et autres (2013). «Leadership as Boundary Work in Healthcare Teams », Leadership, vol. 9, no 2, p. 201-228. 
Chreim, S. et autres (2010). "Change Agency in a Primary Health Care Context: The Case of Distributed Leadership », Health Care Management Review, vol. 35, n² 2, p. 187-199.

Centre de réadaptation Marie Enfant (2013). Notre mission, www.crme-sainte-justine.org/ pages.aspx?IdMenu=1763\&IdPage=40002 (page consultée le 15 janvier 2013).

Centre de réadaptation Marie Enfant (2009). La Fondation Marie Enfant devient la Fondation Mélio!, www.crme-sainte-justine.org/nouvelle.aspx?id nouvelles=51800 (page consultée le 13 février 2013).

Clark, S. M. et autres (2010). «Transitional Identity as a Facilitator of Organizational Change During a Merger », Administrative Science Quarterly, vol. 55, n 3, p. 397-438.

Comtois, E., J.-L. Denis et A. Langley (2004). «Rhetorics of Efficiency, Fashion and Politics: Hospital Mergers in Quebec », Management Learning, vol. 35, n 3, p. 303-320.

Denis, J.-L. et autres (2009). «The Reciprocal Dynamics of Organizing and Sense-Making in the Implementation of Major Public-Sector Reforms ", Administration publique du Canada, vol. 52, no 2 , p. 225-248.

Denis, J.-L. et autres (1999). "The Struggle to Redefine Boundaries in Health Care Systems », dans D. Brock, M. Powell et C. R. Hinings (dir.), Restructuring the Professional Organization, Routledge, London, p. 105-130.

Denis, J.-L., L. Lamothe et A. Langley (2006). « Reforming Health Care: Levers and Catalysts for Change », dans A. L. Casebeer et autres (dir.), Innovations in Health Care: A Reality Check, Palgrave Macmillan, Royaume-Uni, p. 3-16.

Denis, J.-L., L. Lamothe et A. Langley (1999). « The Struggle to Implement Teaching Hospital Mergers », Canadian Public Administration, vol. 42, no 3, p. 286-311.

Forcioli, P. (2002). Rapport du groupe d'étude et de recherche sur les fusions et regroupements d'établissements publics de santé, Fédération hospitalière de France, Groupe d'étude et de recherche sur les regroupements et fusions d'établissements publics de santé.

Fulop, N. et autres (2005). «Changing Organisations: A Study of the Context and Processes of Health Care Providers in England », Social Science and Medicine, vol. 60, n 1, p. 119-130.

Heracleous, L. (2004). "Boundaries in the Study of Organization», Human Relations, vol. 57, $\mathrm{n}^{\circ}$ 1, p. 95-103.

Hernes, T. (2003). «Enabling and Constraining Properties of Organizational Boundaries », dans N. Paulsen et T. Hernes (dir.), Managing Boundaries in Organizations: Multiple Perspectives, Palgrave Macmillan, New York, p. 35-54.

Hernes, T. et N. Paulsen (2003). "Introduction: Boundaries and Organization », dans N. Paulsen et T. Hernes (dir.), Managing Boundaries in Organizations: Multiple Perspectives, Palgrave Macmillan, New York, p. 1-13.

Kitchener, M. et L. Gask (2003). " NPM Merger Mania: Lessons from an Early Case », Public Management Review, vol. 5, no 1, p. 20-44.

Langley, A. (1999). "Strategies for Theorizing form Process Data », Academy of Management Review, vol. 24, no 4, p. 691-713.

Langley, A. et autres (2012). "Identity Struggles in Merging Organizations: Renegotiating the Sameness-Difference Dialectic», The Journal of Applied Behavioral Science, vol. 48, n² p. 135-167. 
Marshall, N. (2003). «Identity and Difference in Complex Projects: Why Boundaries still Matter in the "Boundaryless" Organization », dans N. Paulsen et T. Hernes (dir.), Managing Boundaries in Organizations: Multiple Perspectives, Palgrave Macmillan, New York, p. 55-75.

Pettigrew, A. M. (1990). «Longitudinal Field Research on Change », Organization Science, vol. 1, $n^{\circ} 3$, p. 267-292.

Raison, M. et autres (2011). "Le génie de la réadaptation pédiatrique au CRME », Le Capteur d'idées, vol. 7, n 1, p. 12-15.

Samson Saulnier, G. (2007). «Les pratiques de réadaptation en constante évolution: Les acquis... Les défis », Le Capteur d'idées, vol. 3, no 1, p. 3-5.

Santos, F. M. et K. M. Eisenhardt (2005). "Organizational Boundaries and Theories of Organization », Organization Science, vol. 16, no 5, p. 491-508.

Vaillancourt, J. (2005). «Un guide de rédaction des programmes de réadaptation », Le Capteur d'idées, vol. 1, n 3, p. 4. 